into the pathophysiology of aneurysm healing and development of more effective therapeutic options. As inflammatory processes appear to be the primary mechanism underlying cerebral aneurysm pathophysiology, it is critical to investigate aneurysm healing in the context of key inflammatory mediators. A more robust understanding of underlying inflammatory processes is crucial to developing novel treatments for complete resolution of unruptured cerebral aneurysms. Using a novel in vitro flow chamber model, we have identified chemokine (C-X-C) motif ligand 1 (CXCL1) as an important mediator in aneurysm pathophysiology. As there is increased expression of CXCL1 at arterial bifurcations and in aneurysms in vitro, we hypothesized that CXCL1 may be a key mediator in aneurysm healing.

Methods Using our murine aneurysm healing model, aneurysms were induced in the right common carotid artery of C57BL/6 mice using elastase. Three weeks later when aneurysm formation was complete, aneurysms were implanted with either poly(lactic-co-glycolic acid)(PLGA)+CXCL1 - coated coils or PLGA only - coated coils. Three weeks after coil implantation, aneurysms were harvested for histological quantification of aneurysm healing. In a subsequent experiment, aneurysms were induced in $\mathrm{C} 57 \mathrm{BL} / 6$ mice using the same elastase model. Three weeks after aneurysm induction, all mice underwent aneurysm coiling with PLGA-coated coils. Animals were randomly assigned to receive intraperitoneal injections of either CXCL1 neutralizing antibody or isotype-matched IgG control. Aneurysm tissue was harvested for histological quantification of aneurysm healing.

Results In the first experiment, we found that animals treated with PLGA+CXCL1 - coated coils had significantly less aneurysm healing than those treated with PLGA only - coated coils $(21.8 \% \pm 3.87$ versus $39.8 \% \pm 8.02$, respectively; $\mathrm{p}=$ 0.048). In the second experiment, animals treated with CXCL1 neutralizing antibody had significantly increased aneurysm healing compared to those treated with IgG control $(63.8 \% \pm 3.69$ versus $42.4 \% \pm 3.55$, respectively; $\mathrm{p}=$ $0.00012)$.

Conclusion Our findings suggest CXCL1 decreases murine aneurysm healing after coil implantation. Therapeutic intervention with CXCL1 neutralizing antibody appears to increase aneurysm healing after coil implantation.

Disclosures D. Patel: None. K. Motwani: None. K. Hosaka: None. B. Hoh: None.

\section{E-016 IN VITRO VASCULAR MODEL MATERIAL CHARACTERIZATION}

M McCue*, W Merritt, C Settanni, T Becker. Bioengineering Devices Laboratory, Mechanical Engineering, Northern Arizona University, Flagstaff, AZ

\subsection{6/neurintsurg-2019-SNIS.91}

Introduction Endovascular devices aid in the treatment of heart failure, thrombus removal, and aneurysms. To assist in the testing and development of new endovascular devices, in vitro training models are used during the proof-of-concept and prototype testing phases of product development. Although current vessel-training models cast from human vasculature are anatomically accurate, the materials often used for casting (i.e. silicones and glass) do not accurately simulate the vascular compliance (modulus), wall friction effects (lubricity), and hardness values seen in native human vasculature. Thus, more flexible and comprehensive in vitro models are needed to better understand how endovascular devices (such as coils, stents, flow diverters, flow disruptors, liquid embolics, and thrombectomy devices delivered via guide catheters, guide wires, and delivery microcatheters) physically interact with the vessel wall and corresponding tissues.

Methods To address the shortcomings of commercially-available vascular flow models, materials were developed and optimized in this study to have similar mechanical properties to human tissue. To accomplish this, both donated 'fresh' cadaveric vascular tissues (common carotid artery) and synthetic biomimetic materials were mechanically characterized with a hybrid rheometer (DH-R 2, TA Instruments - Figure 1).

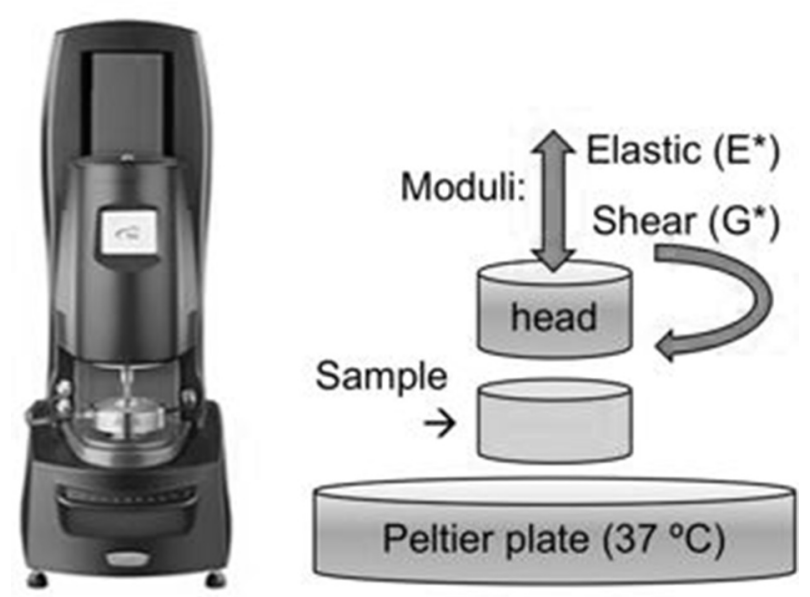

Abstract E-016 Figure 1 Left - Hybrid Rheometer. Right Rheometer Setup for Sample Modulus

Results Via the rheometer, data was collected for luminal wall friction, radial compliance, shear modulus $\left(G^{*}\right)$, and elastic modulus $\left(\mathrm{E}^{*}\right)$. Properties of cadaveric vessels and model materials were statistically compared, and the biomaterials were tuned to closely mimic the mechanical properties of the cadaveric vasculature. The biomaterials were manufactured into flow models. 3D printing manufacturing techniques were used to obtain repeatable anatomical accuracy. Validation of the models by partnered neurointerventionalists is underway to ensure realistic catheter trackability and anatomical accuracy.

Discussion The new biomimetic materials in this study were able to simulate the compliance and mechanical properties of human vasculature more closely than existing silicone, polyurethane, and glass models. The utilization of novel biomimetic materials within this in vitro vascular flow model will allow for more relevant benchtop testing of endovascular devices. These models have the potential to generate more accurate data on device performance and may reduce the need for costly in vivo studies.

Disclosures M. McCue: 1; C; Brain Aneurysm Foundation Grant-2018. W. Merritt: 1; C; Brain Aneurysm Foundation Grant-2018. 5; C; Northern Arizona University. C. Settanni: 1; C; Brain Aneurysm Foundation Grant-2018. 5; C; Northern Arizona University. T. Becker: 1; C; Brain Aneurysm Foundation Grant-2018. 5; C; Northern Arizona University, Aneuvas Technologies, Inc. 